\title{
Amorphization in zircon: evidence for direct impact damage
}

\author{
S Ríos†§, E K H Salje†, M Zhang† and R C Ewing \\ $\dagger$ Department of Earth Sciences, University of Cambridge, Downing Street, \\ Cambridge CB2 3EQ, UK \\ \$Department of Nuclear Engineering and Radiological Sciences, University of Michigan, \\ Ann Arbor, MI 48109-2104, USA
}

Received 26 November 1999, in final form 2 February 2000

\begin{abstract}
X-ray diffraction has been used to characterize the amorphous phase present in a series of radiation-damaged natural zircons with radiation doses ranging from 0.06 to $16 \times 10^{18}$ $\alpha$-decay events $\mathrm{g}^{-1}$. The fraction of amorphous material present in each of the samples studied has been determined, and its dependence on the radiation dose has been calibrated. Direct determination of the amorphous fraction confirms that amorphization in natural zircon occurs as a consequence of the direct impact within cascades caused by $\alpha$-recoil nuclei. These results are not consistent with the commonly accepted double-overlap model of damage accumulation.

The volume swelling of amorphous regions changes as a function of dose. Thus, the density of amorphous regions depends on the degree of damage up to a certain point (i.e. $8 \times 10^{18}$ $\alpha$-decay events $\mathrm{g}^{-1}$ ), unlike in previous models for which a constant value independent of the radiation dose was assumed.
\end{abstract}

\section{Introduction}

Naturally occurring phases such as zircon $\left(\mathrm{ZrSiO}_{4}\right)$, titanite $\left(\mathrm{CaTiSiO}_{5}\right)$, the apatites $\left(\mathrm{Ca}_{10}\left(\mathrm{PO}_{4}\right)_{6}(\mathrm{OH}, \mathrm{Cl}, \mathrm{F})_{2}\right)$ and pyrochlores $\left(\mathrm{A}_{1, \ldots, m} \mathrm{~B}_{2} \mathrm{O}_{6}(\mathrm{O}, \mathrm{OH}, \mathrm{F})_{1, \ldots, n} \cdot \mathrm{pH}_{2}\right.$ with $\mathrm{A}=\mathrm{Na}$, $\mathrm{Ca}, \mathrm{RE}, \ldots$ and $\mathrm{B}=\mathrm{Nb}, \mathrm{Ta}, \ldots)$ are known to undergo amorphization as a consequence of the $\alpha$-decay of radionuclide impurities (typically ${ }^{238} \mathrm{U},{ }^{235} \mathrm{U}$ and ${ }^{232} \mathrm{Th}$ and their decay products) (Holland and Gottfried 1955, Lumpkin and Ewing 1988, Ewing 1994, Weber et al 1994). Due to the properties (mainly chemical durability) that some of these materials offer as waste forms for the immobilization of high-level waste and plutonium (Weber et al 1998, Ewing 1999), natural materials and their synthetic equivalents have been extensively studied. In order to elucidate the amorphization process, the effect of natural radioactive impurities has been simulated by heavy-ion bombardment (e.g. $\mathrm{Kr}^{+}, \mathrm{Xe}^{+}$) (Wang and Ewing 1992a, Meldrum et al 1996) or by actinide doping $\left({ }^{238} \mathrm{Pu},{ }^{244} \mathrm{Cm}\right)$ (Weber 1990, Weber et al 1994, 1997b).

During the amorphization process in natural zircon three different stages may be observed with increasing dose, depending on the age of the sample and/or the content of radioactive impurities (Holland and Gottfried 1955, Murakami et al 1986, 1991). At low degrees of damage the material is essentially crystalline, $\alpha$-particles produce isolated defects and $\alpha$-recoil nuclei produce a few isolated amorphous regions. At this first stage, sharp Bragg maxima are observed that decrease in intensity with increasing radiation dose. The crystalline matrix is expanded (as shown by the unit-cell swelling (Holland and Gottfried 1955, Murakami et al 1991)) as a consequence of the shear deformation produced by the localized defects created by $\alpha$-particles

$\S$ Author to whom any correspondence should be addressed. 
(Ríos and Salje 1999). At intermediate doses the decrease of the intensity of diffraction maxima and their broadening is accompanied by the appearance of two diffuse rings, as observed from electron diffraction images: in natural (Murakami et al 1991, Capitani et al 2000) and irradiated zircon (Wang and Ewing 1992b, Weber et al 1994) samples, pyrochlores (Lumpkin and Ewing 1988) and Cm-doped $\mathrm{Ca}_{2} \mathrm{Nd}_{8}\left(\mathrm{SiO}_{4}\right) \mathrm{O}_{2}$ (Weber 1993), for example. These rings, which are due to the increasing content of aperiodic regions in the material, become clearly visible at the final stage of the amorphization process, when most of the material is amorphous. No sharp diffraction maxima are detectable at this third stage, indicating the loss of long-range order. These diffuse haloes evidence the remaining short-range order that persists in the fully amorphous phase. The transformation undergone by radiation-damaged zircon has recently been interpreted in terms of a percolation-type transition rather than as anorphization phase transition (Salje et al 1999), where at the percolation point the less abundant state (the aperiodic material at low doses, and the crystalline material at high doses) forms an interconnected network.

The goal of the present work is to identify the properties of the aperiodic phase created during the amorphization process. The relevant questions are: firstly, what is the amount of amorphous material produced by each of the $\alpha$-recoil nuclei, secondly, what is the fraction of amorphous material in natural zircon as a function of $\alpha$-decay-event radiation doses and, thirdly, is the density of the amorphous cascade independent of the dose, or does it change with the degree of damage? These questions have already been addressed previously (Weber 1993, Weber et al 1994), although in those cases the authors started from the assumption that the swelling of amorphous regions was independent of the radiation dose and equal to approximately $18 \%$ - the maximum macroscopic swelling observed in zircon.

Models of the data in this paper show that this assumption is not correct and that, consequently, several of the previous proposals for the damage mechanism require revision. We will argue that the direct amorphization model (Gibbons 1972) satisfactorily explains the amorphization process in natural zircon, with no need to turn to the more elaborate overlap model (Gibbons 1972, Carter and Webb 1979).

\section{Experimental procedure}

A series of natural zircon samples with different degrees of radiation damage were used for this study (table 1). All samples originate from Sri Lanka, and have different colours and morphologies. The major impurity present in all of them (apart from $\mathrm{U}$ and $\mathrm{Th}$ ) is hafnium at less than $2.2 \mathrm{wt} \%$. Although some of the highly damaged zircon samples do still have crystal faces, none of the samples reported in this work possess a well defined morphology. Flat surfaces present on the samples were used for the diffraction experiment. Their sizes varied between 1 and $5 \mathrm{~mm}$ in the longest direction.

The diffractometer used for this study has been described previously (Locherer et al 1996). It has four degrees of freedom like a standard four-circle diffractometer and, in addition, means for controlled $x$ - and $y$-translation of the goniometer cradle. These two extra degrees of freedom are essential for testing the homogeneity of the samples. Copper $\mathrm{K} \alpha_{1}$ x-rays produced by the fixed-anode generator pass through a set of collimator slits onto a focusing monochromator and two further sets of slits to the sample. The final slits were fixed to $0.5 \mathrm{~mm}$ width and $0.5 \mathrm{~mm}$ height. The sample height was adjusted each time as accurately as possible in order to reduce the shift in the $2 \theta$ position of the Bragg peaks from one sample to another. The position-sensitive linear detector covers a $2 \theta$ range of $120^{\circ}$, with an angular resolution of about $0.03^{\circ}$. In order to decrease the intensity of the background signal coming mainly from the air scattering, the collimation between monochromator and sample was extended to 
Table 1. $\alpha$-decay-event doses of radiation-damaged zircons used for this study.

\begin{tabular}{lll}
\hline Sample & Dose $\left(10^{18} \alpha\right.$-decay events $\left.\mathrm{g}^{-1}\right)$ & Reference \\
\hline 4403 & 0.06 & Murakami et al $(1991)$ \\
269 & 1.8 & Zhang et al $(2000)$ \\
4303 & 2.1 & Murakami et al $(1991)$ \\
Cam26 & 2.9 & Zhang et al $(2000)$ \\
Z5 & 4.2 & Zhang et al $(2000)$ \\
S4 & 4.8 & Zhang et al $(2000)$ \\
4304 & 5.8 & Murakami et al $(1991)$ \\
4105 & 6.3 & Murakami et al $(1991)$ \\
3107 & 7.2 & Ellsworth et al $(1994)$ \\
Z2 & 8.6 & Zhang et al $(2000)$ \\
Ti8 & 9.6 & Zhang et al $(2000)$ \\
6500 & 11.7 & Woodhead et al $(1991)$ \\
157 & 13.1 & Zhang et al $(2000)$ \\
Sd4 & 15.9 & Zhang et al $(2000)$ \\
\hline
\end{tabular}

as close as possible to the sample. As our experiment was mainly focused on the very weak intensity arising from the amorphous areas created by radiation damage, and not on the Bragg reflections themselves, long counting times of 18-24 hours per sample position were needed in order to reduce the statistical noise of the diffractograms. Samples were mounted with no specific orientation, as the scattered intensity from the amorphous areas is expected to be isotropically distributed. Rocking experiments were performed on each of the samples in order to check the possible orientation dependence of the diffraction signal.

\section{Results}

Figure 1 shows a typical diffractogram obtained from a weakly damaged zircon (sample 4403, radiation dose about $0.06 \times 10^{18} \alpha$-decay events $\mathrm{g}^{-1}$ ). For this particular rocking position, we observe two well defined Bragg reflections, (301) and (600), against a flat unstructured background, as is expected from the very low degree of radiation damage of the crystal. The high intensity at low scattering angles, indicated by a dotted line in figure 1, originates from air scattering. All graphs have been normalized to the same counting time, and the air scattering subtracted.

In samples with higher degrees of damage, broad Bragg reflections are still visible. Simultaneously the background starts to show some broad and weak ripples. Figure 2 shows the diffractograms obtained from three different zircon samples: 4403 (low dose), 269 (intermediate dose) and Ti8 (high dose). For the particular rocking angle chosen for this graph, sample 269 has two atomic planes in the diffraction positions (112) (at about $2.5 \AA^{-1}$ and indicated by a dotted line in figure 2) and (613) (at about $5.8 \AA^{-1}$ ). The two broad peaks observed for sample 269-figure 2-and indicated by a continuous line and an arrow respectively, are related to the amorphous phase already present in the crystal. Sample 269 contains some $15 \%$ of amorphous regions (see below in the text). The identification of the signals of the amorphous phase also relates to the isotropic nature of the diffraction. The maximum at $4 \AA^{-1}$, for example, does not show any orientation dependence in a large rocking scan (see figure 3 ) - in contrast to the neighbouring peaks that are due to Bragg reflections associated with the crystalline areas. The inset of figure 2 shows the large increase in the 


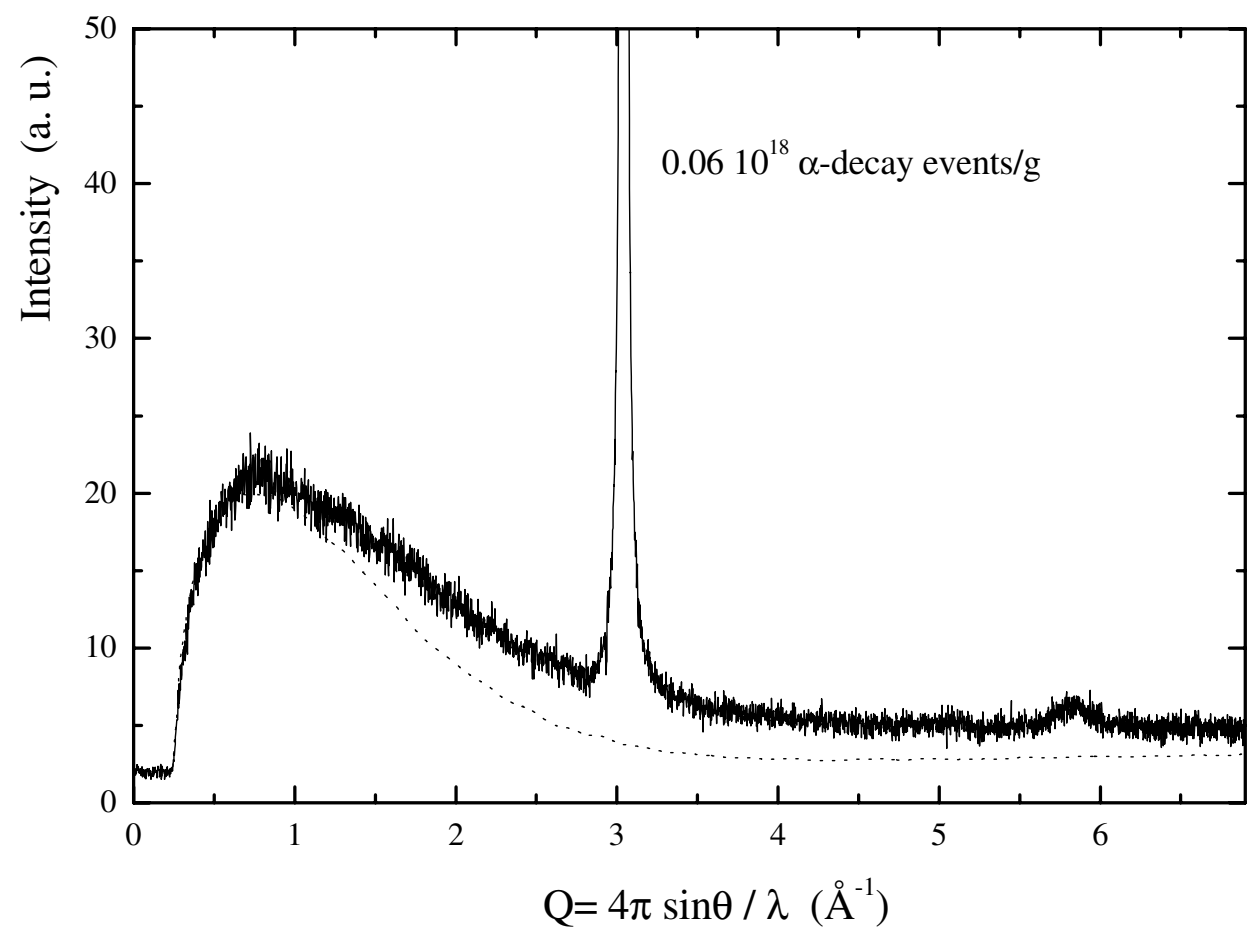

Figure 1. A diffractogram obtained for a particular rocking position of sample 4403. Two Bragg reflections are visible: (301) and (600). The dotted line indicates the intensity arising from the air scattering. The intensity in each diffractogram has been normalized to one hour of counting time.

background intensity at high $2 \theta$ values as a consequence of the increasing fraction of amorphous areas in the sample. The dotted and continuous lines in figure 2 and figure 3 indicate the two Gaussian contributions for the first peak of the diffractogram of sample 269, originating from a Bragg reflection and the amorphous phase, respectively.

No Bragg peaks are evident in the patterns for samples with high radiation doses $\left(>8 \times 10^{18} \alpha\right.$-decay events $\left.\mathrm{g}^{-1}\right)$ - figure 2 -while four well defined broad peaks characterize the diffraction pattern of zircon. The strongest is at $2 \AA^{-1}$, there are two at about 3.5 and $3.9 \AA^{-1}$ and the weakest is at around $6 \AA^{-1}$. This profile matches very well with the diffraction pattern previously reported and obtained by electron diffraction (Murakami et al 1991, McLaren et al 1994, Capitani et al 2000). In the latter measurements the first ring was at $2.0 \AA^{-1}$, which agrees with our first peak. Their second ring is very close to the average position of our second and third peaks (3.5 and $\left.3.9 \AA^{-1}\right)$. Previous electron diffraction measurements were not able to resolve these two latter peaks. The weak hump at high $Q$-values, near $6 \AA^{-1}$, was observed in some of the electron diffraction measurements - for example, those of Weber (1993).

All samples with high degrees of radiation dose (Ti8, 6500, 157, Sd4) show the same basic features-see figure 4-with no significant dependence on the dose. All four samples appear to consist of one unique phase, namely the aperiodic phase, and no extra peaks are observed. Only sample 157 (radiation dose $13.1 \times 10^{18} \alpha$-decay events $\mathrm{g}^{-1}$ ) shows an extra peak, indicated by the arrow in figure 4, which may be due to small amounts of $\mathrm{ZrO}_{2}$. This crystal is known to be heavily damaged, containing one unique phase, as shown by Raman spectroscopic measurements (Zhang et al 2000). However, the presence of some zirconia grains in this particular specimen suggests previous thermal annealing. 


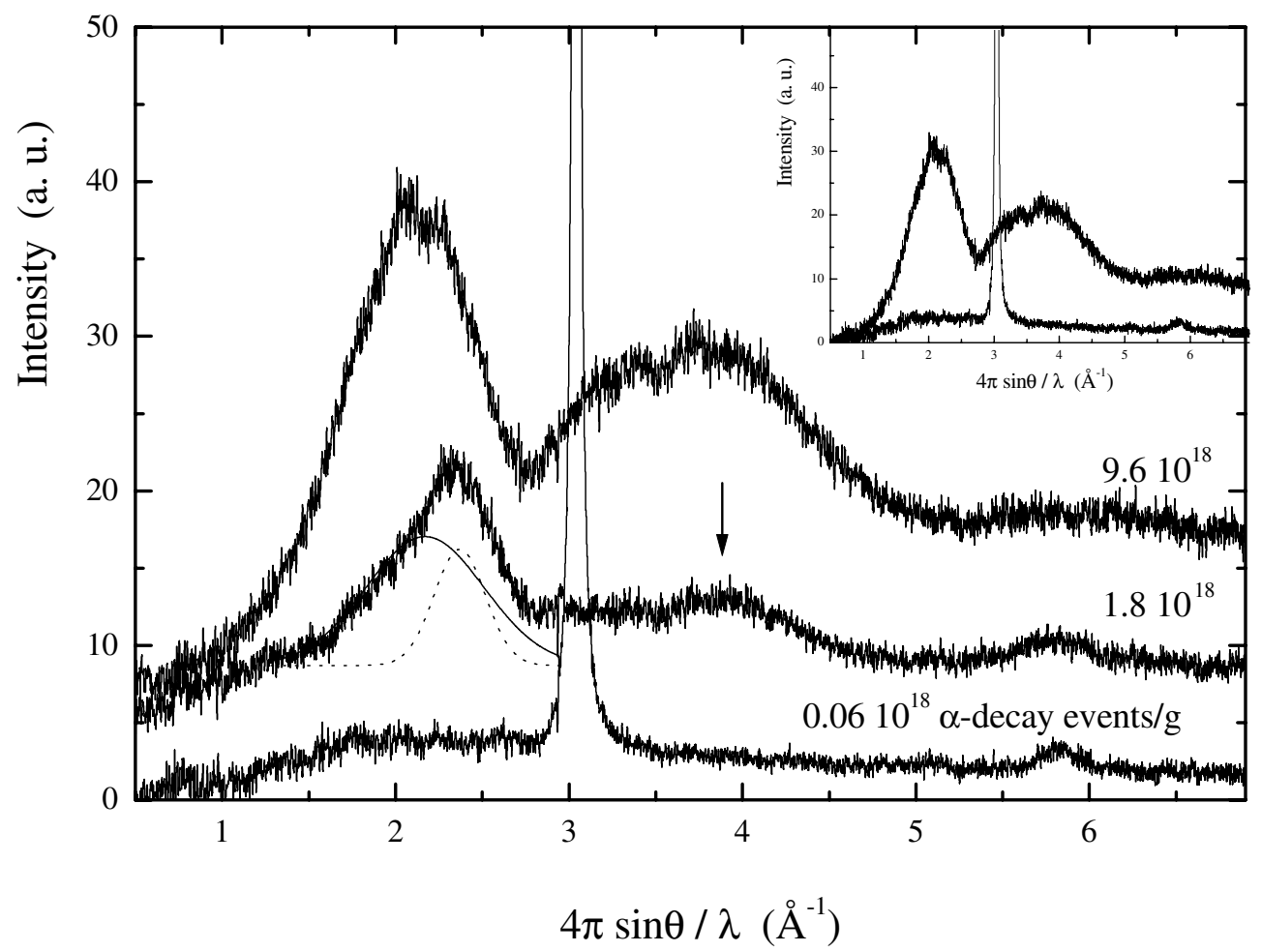

Figure 2. Diffractograms obtained for samples 4403, 269 and Ti8. For clarity, the diffractograms have been shifted upwards. In the inset, the increase of the background with increasing radiation damage due to the higher fraction of amorphous areas is evident. The first peak for sample 269, at around $2.5 \AA^{-1}$, has two contributions: the first one due to crystalline areas (dotted line) and the second one due to amorphous areas (continuous line). The peak indicated by an arrow is associated with aperiodic regions.

\section{Discussion}

\subsection{Amorphization process}

From the intensity scattered by the amorphous regions, we can estimate the fraction of amorphous material present in each sample. As shown in figure 5, the intensity for large scattering vectors increases monotonically with increasing radiation dose. As the intensity scattered by the amorphous regions is proportional to the fraction present in the sample, the data can be directly used for comparison with the fraction of amorphous material, $f_{a}$, obtained previously (Weber 1990, 1993, Weber et al 1994).

In order to make the comparison, the fraction of amorphous volume determined experimentally from our measurements was defined as follows:

$$
f_{a}^{\text {exp }}(\text { sample })=\left(I_{\text {sample }}-I_{4403}\right) / I_{\text {Sd } 4}
$$

where $I_{4403}, I_{\mathrm{Sd} 4}$ and $I_{\text {sample }}$ are the intensities scattered by samples $4403, \mathrm{Sd} 4$ and any sample studied, respectively. In a first attempt, $I_{\text {sample }}$ was chosen to be the intensity integrated over the whole $Q$-range, after the background and the Bragg reflections had been subtracted. However, the data showed quite a significant scatter, which made the analysis difficult. The scatter of the data is basically due to the fact that the relative intensity of the first two broad peaks depends 


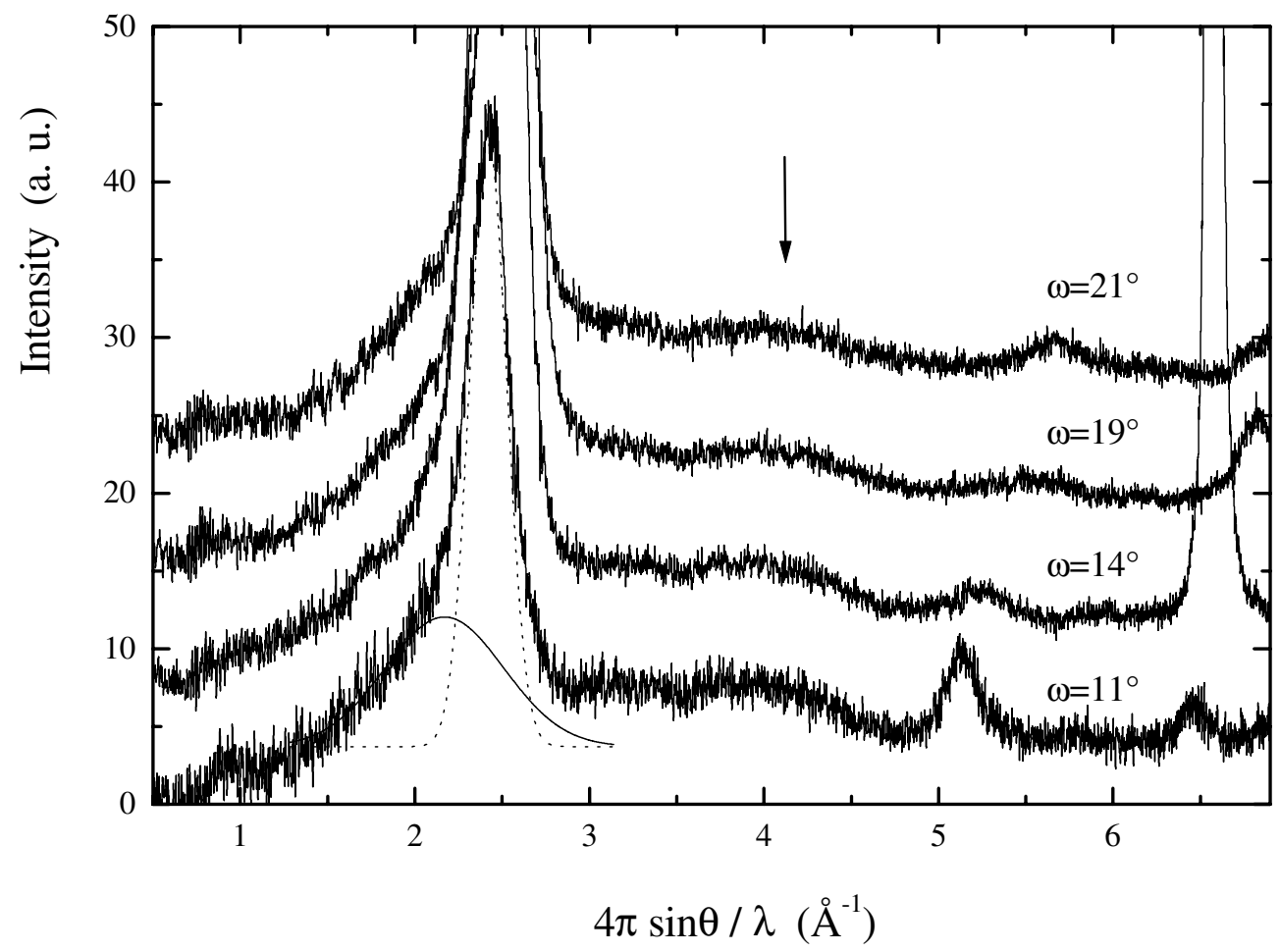

Figure 3. The angular dependence of the diffractograms for sample 269 (dose $1.8 \times 10^{18} \alpha$-decay events $\mathrm{g}^{-1}$ ). The diffractograms were obtained at rocking angles of (starting from the bottom) $11^{\circ}, 14^{\circ}, 19^{\circ}$ and $21^{\circ}$. They have all been shifted upwards for clarity. The broad peak indicated by an arrow, and which can be observed in all the diffractograms, is related to amorphous areas present in the crystal. While the sharp peaks are arising from crystalline areas, the first peak, at around $2.5 \AA^{-1}$, has two contributions: from crystalline areas (dotted line) and amorphous areas (continuous line).

strongly on two factors: (i) the roughness of the sample surface; and (ii) the angle between the sample and the incoming beam, as could be seen from the rocking scans. While the intensity in the $1<Q<5 \AA^{-1}$ range for a particular sample appears to be affected by these two factors, the intensity for $Q>5 \AA^{-1}$ proved to be rather constant. Therefore, as the above-mentioned effects are difficult to quantify, $I_{\text {sample }}$ was chosen at $Q>5 \AA^{-1}$ (where no strong peaks are present); in particular, $Q_{0}=6.5 \AA^{-1}$. The amorphous fractions as determined from our data, $f_{a}^{e x p}$, are shown in figure 6 as full circles. These data have proved to be in very good agreement with the amorphous fraction obtained by infrared spectroscopy measurements (to be published elsewhere), shown in figure 6 as open circles.

Four different amorphization mechanisms have been reported in the literature as regards materials susceptible to amorphization:

(i) due to the accumulation of point defects (Gong et al 1996),

(ii) by interface-controlled amorphization (Motta 1997),

(iii) by multiple cascade overlap (Gibbons 1972) and

(iv) by in-cascade amorphization (Weber 1993).

In some materials, the amorphization is in fact a combination of these processes (Carter 1983, Lindner 1996). Moreover, amorphization is known to be strongly dependent on parameters 


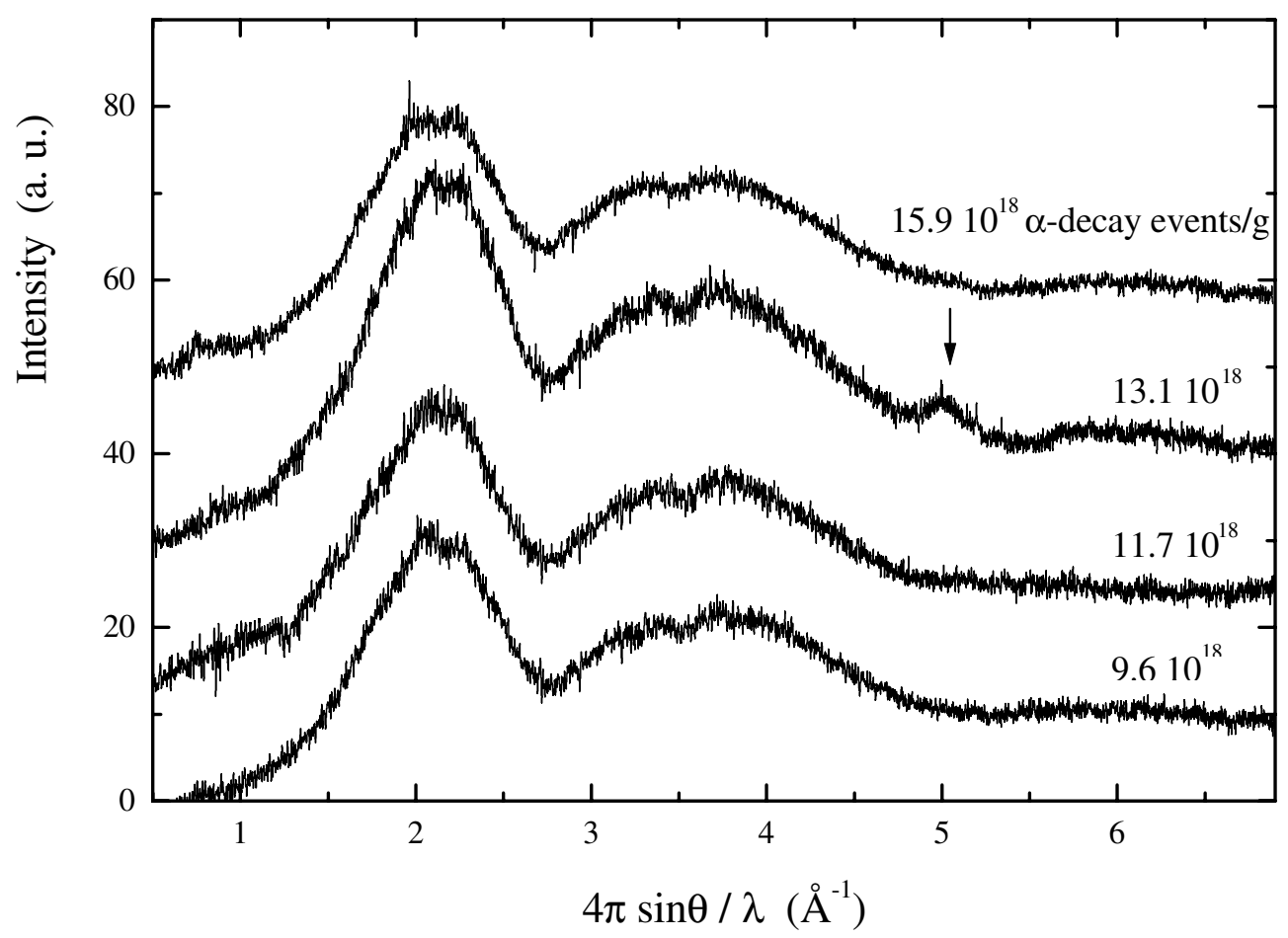

Figure 4. Diffractograms obtained for four heavily damaged samples: Ti8, 6500, 157 and Sd4. All show basically the same features. They have been shifted upwards for clarity. The extra peak for 157 , and indicated by an arrow, is due to recrystallized $\mathrm{ZrO}_{2}$.

such as temperature (Webb and Carter 1981, Weber 2000) and crystallization efficiency (Wang et al 1998), as recovery processes can reduce the damage production. The amorphous fraction as a function of the degree of damage varies in each of the above processes (Gibbons 1972). Thus, the amorphous fraction can be used to obtain insight into the amorphization process. For the particular case of natural zircon, amorphous cascades due to $\alpha$-recoil nuclei are known to be the main mechanism of destruction of crystallinity, as can be seen from the existence of amorphous regions even in the early stages of the amorphization process. However, the mechanism appears not to be identical for all materials. In coesite, for example, amorphization occurs as a consequence of point defect accumulation (Gong et al 1996). The question now is whether amorphization in natural zircon is created within the cascades originating from the $\alpha$-recoil nucleus, or only after a certain degree of damage has been reached and, subsequently, damaged regions overlap.

As reported above, the fraction of amorphous material created in natural zircon has been carefully calibrated in this study, and thus we can now compare the observed fraction with established theoretical models - in particular with the direct impact model and the doubleoverlap model (Gibbons 1972). Our experimental data are in very good agreement with the direct impact model (see figure 6), where the amorphization is directly created within the displacement cascade. For such a model, the amorphous fraction is described by the following expression as a function of radiation dose, $D$ :

$$
f_{a}=1-\mathrm{e}^{-B_{a} D}
$$

where $B_{a}$ is the amount of amorphous material produced per $\alpha$-recoil. The dotted line in figure 6 


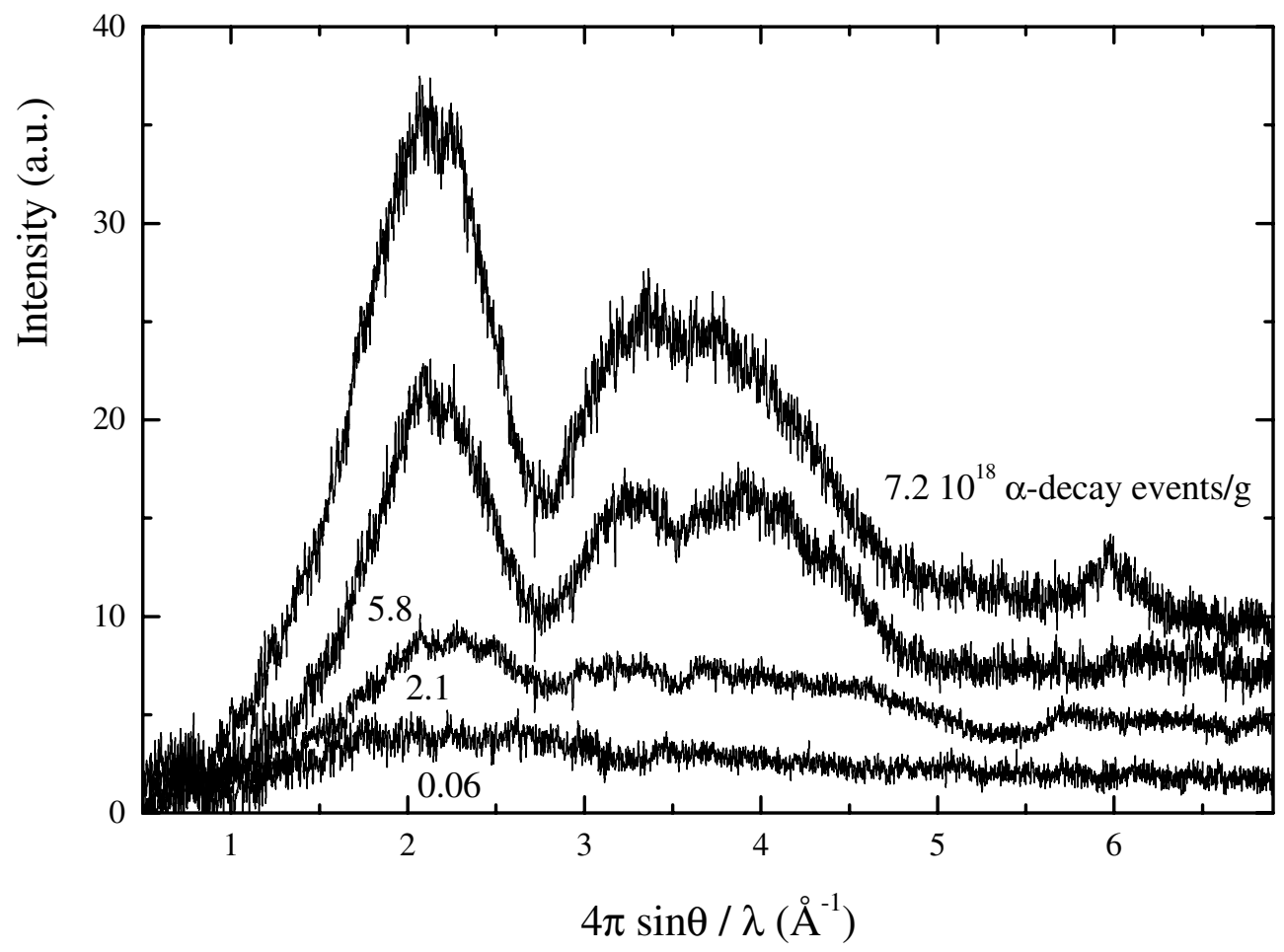

Figure 5. Diffractograms of samples (from the bottom to the top) 4403, 4303, 4304 and 3107. The numbers in the figure indicate the corresponding doses in units of $10^{18} \alpha$-decay events $\mathrm{g}^{-1}$. For the first three samples any contribution from crystalline areas has been removed. For the last sample (3107), which has a high degree of radiation damage, crystalline areas could still be seen, as the peak at $6 \AA^{-1}$ demonstrates. Notice the background increase that occurs when increasing the dose.

is the result of the best fit of equation (1) to our data, which yields a value $B_{a}=2.7(3) \times 10^{-19} \mathrm{~g}$. Thus, the amorphous regions have radii of $\approx 25 \AA$, which is in agreement with the size found by transmission electron microscopy (Weber et al 1994).

Notice the discrepancy in figure 6 between our experimental results and the amorphous fraction derived by Weber et al (1994) (continuous line). In this work, the amorphous fraction was not directly measured, but derived by using the following expression (Weber 1990):

$$
\Delta V_{m} / V_{0}=f_{c} \Delta V_{u c} / V_{0}+f_{a} \Delta V_{a} / V_{0}
$$

which describes the macroscopic swelling observed in zircon as the contributions of the swelling of crystalline and amorphous regions. In equation (2), $\Delta V_{m} / V_{0}$ and $\Delta V_{u c} / V_{0}$ are the experimental macroscopic and unit-cell swelling, respectively, which depend on the radiation dose. $\Delta V_{a} / V_{0}$ is the swelling of the amorphous areas, which was supposed to be constant and equal to $18.4 \%$ - the maximum macroscopic swelling observed in zircon. Furthermore, the condition $f_{a}+f_{c}=1$ holds. The experimental data (optical and density data) given by Holland and Gottfried (1955) were used in equation (2) for $\Delta V_{m} / V_{0}$ and $\Delta V_{u c} / V_{0}$ and, then, the amorphous fraction was fitted using the double-overlap model (Gibbons 1972):

$$
f_{a}=1-\left[\left(1+B_{d} D+\frac{1}{2} B_{d}^{2} D^{2}\right) \mathrm{e}^{-B_{d} D}\right]
$$

with $B_{d}$ the total mass of disordered or damaged material. The continuous line in figure 6 is 


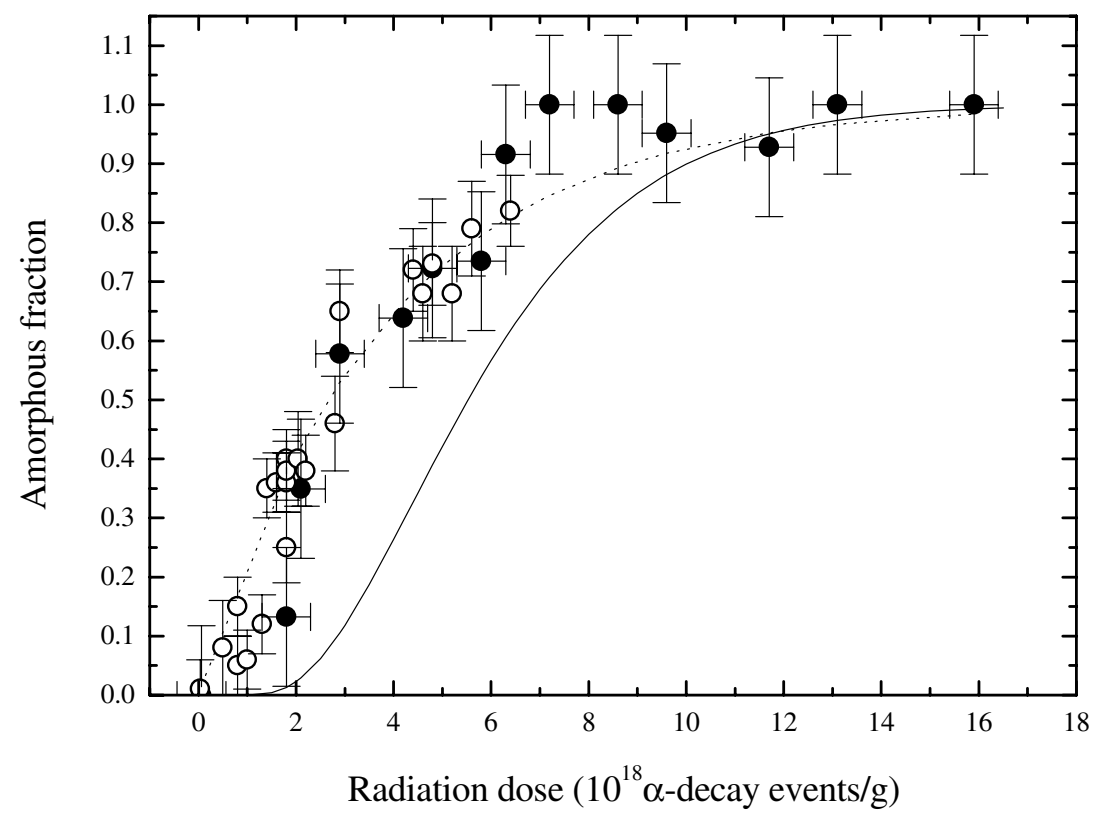

Figure 6. Full circles represent the experimental amorphous fraction, $f_{a}^{\text {exp }}$ as determined following the expression given in the text, versus radiation dose for each of the samples studied; the dotted line shows the best fit obtained using the direct amorphization model. Open circles indicate data from infrared spectroscopy (to be published). $f_{a}$, indicated by a continuous line, is the result of equation (3) as obtained by Weber (1993). The error bars for the dose are estimated to be about $\pm 0.5 \times 10^{18} \alpha$-decay events $\mathrm{g}^{-1}$.

$f_{a}$ as obtained by Weber (1993), with $B_{d}=5.89 \times 10^{-19} \mathrm{~g}$. In this model, the amorphization is only produced after a certain number of damaged regions have been created and begin to overlap; this is the reason for the curvature of $f_{a}$ at low radiation doses. Using this model, an incubation dose of $D_{0}=0.983 \times 10^{18} \alpha$-decay events $\mathrm{g}^{-1}$ had to be assumed in order to get a good agreement between the model and the experimental data in the low-dose range. Using the correct amorphous fraction, as determined in this study, the direct impact model already provides very good agreement, and there is no need to assume an incubation dose or complex overlap behaviour.

\subsection{Swelling of amorphous areas}

As the fraction of amorphous volume contained in a particular sample has been determined in a direct way, we may now use $f_{a}^{\text {exp }}$ and equation (2) to estimate the actual volume swelling of these areas, $\Delta V_{a} / V_{0}$. In equation (2), the values for the macroscopic swelling, $\Delta V_{m} / V_{0}$, and the unit-cell swelling, $\Delta V_{u c} / V_{0}$, were introduced using the analytical expressions derived by Weber (1993). In this work, both are expressed by sigmoidal functions:

$$
\frac{\Delta V_{m, u c}}{V_{0}}=A_{m, u c}\left[1-\mathrm{e}^{-\left(B_{m, u c} D\right)^{n}}\right]
$$

with $A_{m}=18.4 \%, B_{m}=1.61 \times 10^{-19} \mathrm{~g}$ and $n=2.1$, and $A_{u c}=5.06 \%, B_{u c}=3.92 \times 10^{-19} \mathrm{~g}$ and $n=2.3$. Figure 7 shows the volume swelling of the amorphous fraction. The swelling of the amorphous domains is not constant, but depends on the degree of damage of the sample. Only at high doses (above $8 \times 10^{18} \alpha$-decay events $\mathrm{g}^{-1}$ ) is the swelling more or less constant, 


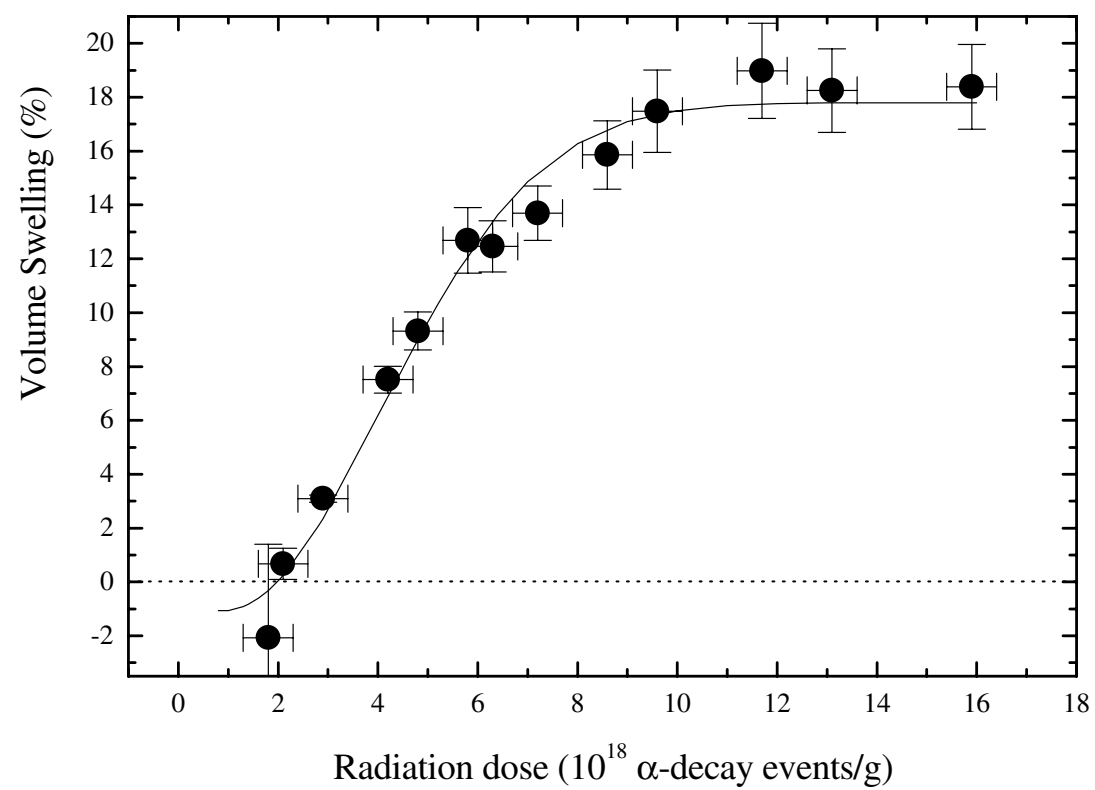

Figure 7. $\Delta V_{a} / V_{0}$ derived using the experimental amorphous fraction obtained in the text and equation (2). The solid line is a guide for the eyes.

equal to some $18 \%$; for lower doses (below $8 \times 10^{18} \alpha$-decay events $\mathrm{g}^{-1}$ ) the density decreases with dose. Furthermore, the swelling of amorphous areas is negative, about $-1 \%$, for radiation doses lower than $2 \times 10^{18} \alpha$-decay events $\mathrm{g}^{-1}$, i.e. the amorphous regions have higher density than the surrounding crystalline material. This could already be anticipated from comparing the experimental macroscopic and unit-cell swellings as shown in figure 11 of Weber (1993). Here, and for the dose range below $2 \times 10^{18} \alpha$-decay events $\mathrm{g}^{-1}$, the unit-cell swelling in natural zircon is shown to be similar to or even larger than the total macroscopic swelling. This, of course, is only possible if amorphous areas have higher density than the defective crystalline regions.

Similar effects have been observed in silica glass (Primak et al 1953) when bombarded with fast neutrons. In this case, the density at low doses is about $3 \%$ greater than that in the unirradiated silica glass, and for higher doses it then gradually decreases to a density $2.7 \%$ larger than that in the original glass. Other nuclear waste glasses, such as alkali silicate glasses, expand at low ion irradiation doses, whereas at high doses they become more compact (Weber et al 1997a).

For the case of zircon, the dependence of the volume swelling of amorphous regions on the dose may be explained as follows. Below the first percolation point $\left(<3 \times 10^{18} \alpha\right.$-decay events $\mathrm{g}^{-1}$ (Salje et al 1999)), when amorphous regions are still isolated and surrounded by the crystalline matrix, the swelling of amorphous domains is small or even negative. At this stage amorphous regions must be compressed by the swelling of the defective expanding crystalline matrix. Above the second percolation point, when the crystalline volume does not percolate any longer and amorphous regions are the majority state, the amorphous regions are free to swell with no constraints imposed by the surrounding material. At this stage (above $8 \times 10^{18} \alpha$-decay events $\mathrm{g}^{-1}$ ) the volume swelling reaches its maximum value, $\approx 18 \%$. Between these two stages, i.e. between the two percolation points, the material contains two types of amorphous volume: 
(i) amorphous regions which have already percolated and

(ii) amorphous regions which are still surrounded by crystalline regions.

Therefore, at this intermediate stage we expect to find amorphous domains having different degrees of swelling: between the minimum value, zero or even negative, and the maximum value of about $18 \%$. In conclusion, amorphous regions found in zircon samples with degrees of damage corresponding to the intermediate and final stages must have slightly different structures. Further studies to determine the structure of the aperiodic regions are in progress.

Two factors which might also be important when studying the swelling of both crystalline and amorphous regions are the impurity content of the sample-mainly hafnium as pointed out above- and the presence of helium bubbles. Nevertheless, helium bubbles are rarely observed in zircon (Headley et al 1981, Weber 1993). Thus, helium accumulation does not seem to be relevant for zircon. The impurity content, on the other hand, varies quite randomly from sample to sample. For example, highly damaged samples may have low (0.4 wt\% in Sd4) or high ( $2.2 \mathrm{wt} \%$ in Ti8) hafnium content. Therefore, and because these impurities are probably distributed in the crystalline as well as in the amorphous regions, their effects on the total swelling are difficult to quantify.

Finally, we comment on the amorphous phase of heavily damaged zircon samples, i.e. for radiation doses above $8 \times 10^{18} \alpha$-decay events $\mathrm{g}^{-1}$. The diffractograms of these samples seem to be basically identical, which is in good agreement with recent results from Raman spectroscopy (Zhang et al 2000). The Si-O stretching modes $\left(1008 \mathrm{~cm}^{-1}\right.$ for the undamaged zircon) for samples Ti8, 6500, 157 and Sd4 show no variation in frequency and linewidth of their corresponding Raman signals. The only difference among these samples seems to be in the fraction of amorphous material, as deduced from the increase in the intensity of the band on increasing the radiation dose. Therefore, we may consider this amorphous phase as the final phase of the complete amorphization process - when almost any atom in the structure has probably been displaced several times by overlapping cascades.

\section{Conclusions}

For the first time, the fraction of amorphous material present in radiation-damaged natural zircon has been directly determined as a function of radiation dose. This direct measurement reveals that amorphization is produced inside the cascade, and that there is no need to use more complex models, such as the double-overlap model, in order to explain the experimental results. Moreover, the aperiodic material is shown to have a volume depending on the degree of damage, which implies that structural differences between different aperiodic states must exist.

\section{Acknowledgments}

The authors wish to thank the following institutions for allowing us to use zircon samples from their collections: the National History Museum of London (UK) for samples 269 (BM.1920,269) and 157 (BM.1921,157); the Sedgwick Museum (University of Cambridge, UK) for Cam26, S4 and Sd4; and the Mineralogisches Museum (Universität Hamburg, Germany) for Z5, Z2 and Ti8. This research project was supported by the European TMR Network contract number FMRX-CT97-0108 (DG12-SLJE) and the US DOE (grant DEFG02-97ER45656 to RCE). 


\section{References}

Capitani G C, Leroux H, Doukhan J C, Ríos S, Zhang M and Salje E K H 2000 Phys. Chem. Minerals submitted Carter G 1983 Radiat. Eff. Lett. 8625

Carter G and Webb R 1979 Radiat. Eff. Lett. 4319

Ellsworth S, Navrotsky A and Ewing R C 1994 Phys. Chem. Minerals 21140

Ewing R C 1994 Nucl. Instrum. Methods Phys. Res. B 9122

Ewing R C 1999 Proc. Natl Acad. Sci. USA 963432

Gibbons J F 1972 Proc. IEEE 601062

Gong W L, Wang L M, Ewing R C and Zhang J 1996 Phys. Rev. B 543800

Headley T J, Ewing R C and Haaker R F 1981 Nature 293449

Holland H D and Gottfried D 1955 Acta Crystallogr. 8291

Lindner J K N 1996 Nucl. Instrum. Methods Phys. Res. B 112316

Locherer K R, Hayward S A, Hirst P J, Chrosch J, Yeadon M, Abell J S and Salje E K H 1996 Phil. Trans. R. Soc. A 3542815

Lumpkin G R and Ewing R C 1988 Phys. Chem. Minerals 162

McLaren A C, Fitzgerald J D and Williams I S 1994 Geochim. Cosmochim. Acta 58993

Meldrum A, Wang L M and Ewing R C 1996 Nucl. Instrum. Methods Phys. Res. B 116220

Motta A T 1997 J. Nucl. Mater. 244227

Murakami T, Chakoumakos B C and Ewing R C 1986 Adv. Ceram.: Nucl. Waste Manage. II 20745

Murakami T, Chakoumakos B C, Ewing R C, Lumpkin G R and Weber W J 1991 Am. Mineral. 761510

Primak W, Fuchs L H and Day P 1953 Phys. Rev. 921064

Ríos S and Salje E K H 1999 J. Phys.: Condens. Matter 118947

Salje E K H, Chrosch J and Ewing R C 1999 Am. Mineral. 841107

Wang L M and Ewing R C 1992a Mater. Res. Soc. Bull. 17538

Wang L M and Ewing R C 1992b Nucl. Instrum. Methods Phys. Res. B 65324

Wang S X, Wang L M, and Ewing R C 1998 Mater. Res. Soc. Symp. Proc. 504165

Webb R P and Carter G 1981 Radiat. Eff. 5969

Weber W J 1990 J. Mater. Res. 52687

Weber W J 1993 J. Am. Ceram. Soc. 761729

Weber W J 2000 Nucl. Instrum. Methods Phys. Res. B at press

Weber W J, Ewing R C, Angell C A, Arnold G W, Cormack A N, Delaye J M, Griscom D L, Hobbs L W, Navrotsky A, Price D L, Stoneham A M and Weinberg M C 1997a J. Mater. Res. 121946

Weber W J, Ewing R C, Catlow R C A, Díaz de la Rubia T, Hobbs L W, Kinoshita C, Matzke H, Motta A T, Nastasi M, Salje E K H, Vance E R and Zinkle S J 1998 J. Mater. Res. 131434

Weber W J, Ewing R C and Meldrum A 1997b J. Nucl. Mater. 250147

Weber W J, Ewing R C and Wang L M 1994 J. Mater. Res. 9688

Woodhead J A, Rossman G R and Silver L T 1991 Am. Mineral. 7674

Zhang M, Salje E K H, Graeme-Marber A, Daniel P, Ewing R C, Clark A M and Leroux H 2000 J. Phys.: Condens. Matter 121915 